\title{
Male fertility influenced by FSHB and FSHR polymorphisms?
}

A genetic polymorphism disrupting transcription of the $\beta$ subunit of folliculestimulating hormone $(\mathrm{FSH}-\beta)$ has an adverse effect on male reproductive parameters. The team of researchers reporting this finding also discovered that men with polymorphisms in the genes encoding FSH- $\beta$ (FSHB) and its receptor FSH-R (FSHR) are more severely affected than those with a mutation in only one of these two genes.

Signalling by FSH through FSH-R is required for development of the testes and for maintaining sperm production during adulthood. Although the FSHR 2039A > G polymorphism has been linked to changes in reproductive parameters in women, no such association has been observed in men. However, the FSHB -211G > T polymorphism has been linked, in men, to reduced serum FSH levels and reduced sperm production.

The researchers analyzed the records of 1,213 men presenting with couple infertility of undefined cause who were treated at the Centre of Reproductive

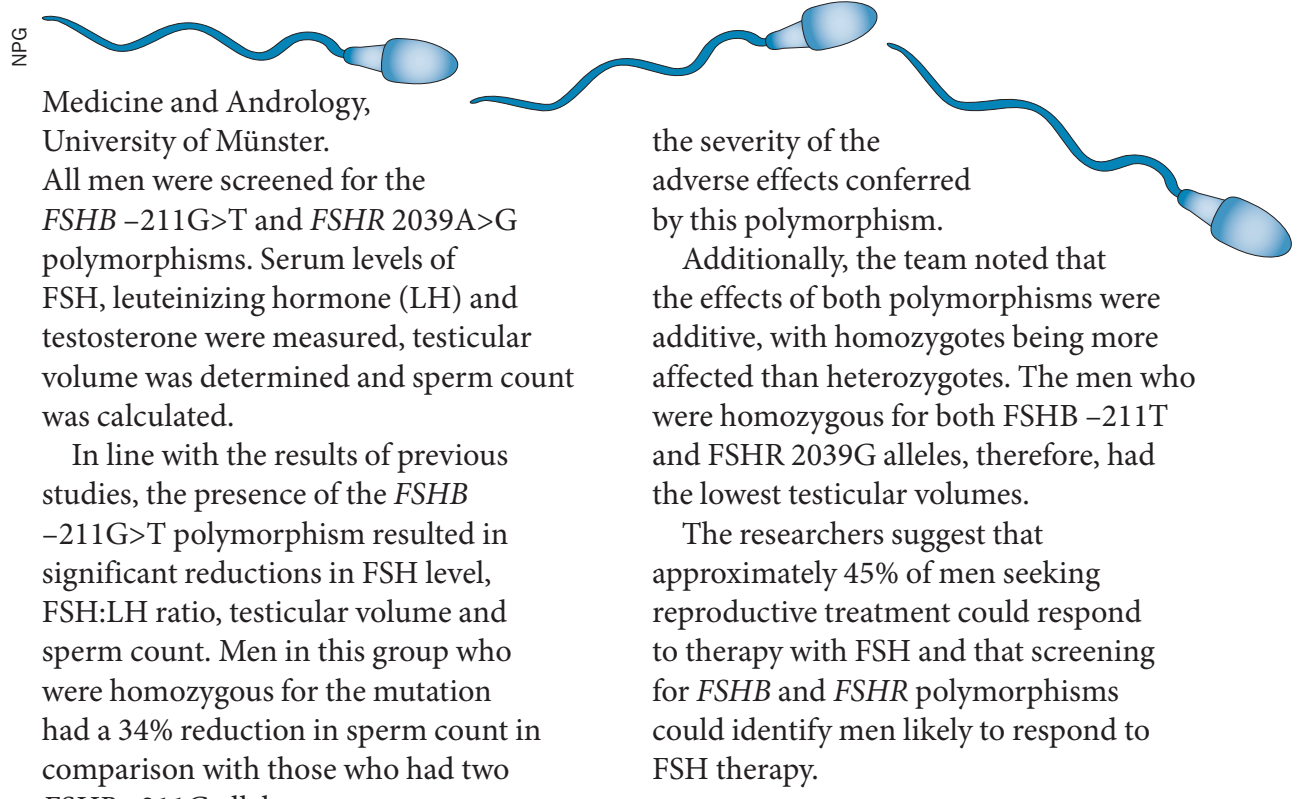

FSHB -211G alleles.

Although no significant associations were observed between FSHR 2039A > G and reproductive parameters, the presence of FSHR 2039G in men with the $F S H B-211 \mathrm{~T}$ polymorphism increased

\section{Fiona Mitchell}

Original article Tüttelmann, F. et al. Combined effects of the variants $F S H B-211 \mathrm{G} / \mathrm{T}$ and $F S H R 2039 \mathrm{~A}>\mathrm{G}$ on male reproductive parameters. J. Clin. Endocrinol. Mebab. doi:10.1210/jc.2012-1761 